\title{
Supplement to "On the sample covariance matrix estimator of reduced effective rank population matrices, with applications to fPCA"
}

\author{
Florentina Bunea*and Luo Xiao ${ }^{\dagger}$
}

January 3, 2014

The following document provides proofs of some of the lemmas, propositions and theorems in [1]. The numbering of lemmas, propositions and theorems will be the same as in [1]. Results in [1] will be used without explicit reference.

\section{A Technical Proofs}

Lemma A.1. Let $X \in \mathbb{R}^{p}$ be a generic vector. Let $\Delta=\left\{u=\left(u_{1}, \ldots, u_{p}\right)^{\prime} \in \mathbb{R}^{p}:\left|u_{1}\right|=\right.$ $\left.\cdots=\left|u_{p}\right|=1\right\}$. Then for any positive integer $d$,

$$
\|X\|^{2 d} \leq \frac{1}{2^{p}} \sum_{u \in \Delta}\left(u^{\prime} X\right)^{2 d} .
$$

*Department of Statistical Science, Cornell University, Ithaca, NY 14850

${ }^{\dagger}$ Department of Biostatistics, Johns Hopkins University, Baltimore, MD 21205 
Poof of Lemma A.1. We write $X$ as $\left(x_{1}, \ldots, x_{p}\right)^{\prime}$. We have

$$
\sum_{u \in \Delta}\left(u^{\prime} X\right)^{2 d}=\sum_{d_{1}+\cdots+d_{p}=2 d} \sum_{u \in \Delta}\left\{\left(\begin{array}{c}
2 d \\
d_{1}, \ldots, d_{p}
\end{array}\right) \prod_{j=1}^{p}\left(u_{j} x_{j}\right)^{d_{j}}\right\} .
$$

It can be shown that

$$
\sum_{u \in \Delta}\left\{\left(\begin{array}{c}
2 d \\
d_{1}, \ldots, d_{p}
\end{array}\right) \prod_{j=1}^{p}\left(u_{j} x_{j}\right)^{d_{j}}\right\}=0
$$

if any of the $d_{j}$ 's is odd. To see this, assume for simplicity that $d_{1}$ is odd. Then

$$
\prod_{j=1}^{p}\left(u_{j} x_{j}\right)^{d_{j}}+\left(-u_{1} x_{1}\right)^{d_{1}} \prod_{j=2}^{p}\left(u_{j} x_{j}\right)^{d_{j}}=0
$$

and $\left(-u_{1}, u_{2}, \ldots, u_{p}\right)$ is also in $\Delta$. It follows that equation (A.1) becomes

$$
\begin{aligned}
\sum_{u \in \Delta}\left(u^{\prime} X\right)^{2 d} & =\sum_{d_{1}+\cdots+d_{p}=d} \sum_{u \in \Delta}\left\{\left(\begin{array}{c}
2 d \\
2 d_{1}, \ldots, 2 d_{p}
\end{array}\right) \prod_{j=1}^{p}\left(u_{j} x_{j}\right)^{2 d_{j}}\right\} \\
& =\sum_{d_{1}+\cdots+d_{p}=d} \sum_{u \in \Delta}\left\{\left(\begin{array}{c}
2 d \\
2 d_{1}, \ldots, 2 d_{p}
\end{array}\right) \prod_{j=1}^{p} x_{j}^{2 d_{j}}\right\} \\
& =2^{p} \sum_{d_{1}+\cdots+d_{p}=d}\left\{\left(\begin{array}{c}
2 d \\
2 d_{1}, \ldots, 2 d_{p}
\end{array}\right) \prod_{j=1}^{p} x_{j}^{2 d_{j}}\right\} \\
& \geq 2^{p} \sum_{d_{1}+\cdots+d_{p}=d}\left\{\left(\begin{array}{c}
d \\
d
\end{array}\right) \prod_{j=1}^{p} x_{j}^{2 d_{j}}\right\} \\
& =2^{p}\|X\|^{2 d}
\end{aligned}
$$

as desired. In the above derivation, we used the inequality

$$
\left(\begin{array}{c}
2 d \\
2 d_{1}, \ldots, 2 d_{p}
\end{array}\right) \geq\left(\begin{array}{c}
d \\
d_{1}, \ldots, d_{p}
\end{array}\right)
$$

which can be easily verified.

Remark A.1. In the following proofs we will assume sometimes, without loss of generality, that $\Sigma$ is a diagonal matrix. This can be immediately justified as follows. Consider the 
eigendecomposition $\Sigma=O D O^{\prime}$, where $O$ is an orthonormal matrix and $D$ is a diagonal matrix. Then $\operatorname{cov}\left(O^{\prime} X\right)=D$ and $\|X\|=\left\|O^{\prime} X\right\|$. Similar arguments can be employed when we consider orthonormal transforms of matrices, and evaluate either their Frobenius or operator norm.

Lemma A.2. Let $X \in \mathbb{R}^{p}$ be a zero-mean sub-Gaussian random vector that satisfies Assumption 1. For any positive integer d,

$$
\mathbb{E}\|X\|^{2 d} \leq \frac{(2 d)^{d}}{c_{0}^{d}}[\operatorname{tr}(\Sigma)]^{d} .
$$

Proof of Lemma A.2. Since $\|X\|^{2 d}$ is invariant under orthonormal transforms, we assume that $\Sigma$ is a diagonal matrix. By Lemma A.1,

$$
\mathbb{E}\|X\|^{2 d} \leq \frac{1}{2^{p}} \sum_{u \in \Delta} \mathbb{E}\left(u^{\prime} X\right)^{2 d}
$$

where $\Delta=\left\{u=\left(u_{1}, \ldots, u_{p}\right)^{\prime} \in \mathbb{R}^{p}:\left|u_{1}\right|=\cdots=\left|u_{p}\right|=1\right\}$. By Assumption 1,

$$
\mathbb{E}\left(u^{\prime} X\right)^{2 d} \leq \frac{(2 d)^{d}}{c_{0}^{d}}\left(u^{\prime} \Sigma u\right)^{d}=\frac{(2 d)^{d}}{c_{0}^{d}}[\operatorname{tr}(\Sigma)]^{d},
$$

where the last equality holds since we assume that $\Sigma$ is a diagonal matrix. It follows that

$$
\mathbb{E}\|X\|^{2 d} \leq \frac{1}{2^{p}} \sum_{u \in \Delta} \frac{(2 d)^{d}}{c_{0}^{d}}[\operatorname{tr}(\Sigma)]^{d}=\frac{(2 d)^{d}}{c_{0}^{d}}[\operatorname{tr}(\Sigma])^{d} .
$$

Lemma A.3. Let $X \in \mathbb{R}^{p}$ be a zero-mean sub-Gaussian random vector and satisfies Assumption 1. Then

$$
\|\| X\|\|_{\psi_{2}}^{2} \leq \frac{2 \operatorname{tr}(\Sigma)}{c_{0}}
$$

Proof of Lemma A.3. Note that $\mathbb{E}\|X\|^{k} \leq \sqrt{\mathbb{E}\|X\|^{2 k}}$ for all $k \geq 1$. Hence by the definition 
of the sub-Gaussian norm and Lemma A.2,

$$
\begin{aligned}
\|\| X\|\|_{\psi_{2}} & =\sup _{k \geq 1} k^{-1 / 2}\left(\mathbb{E}\|X\|^{k}\right)^{1 / k} \\
& \leq \sup _{k \geq 1} k^{-1 / 2}\left(\mathbb{E}\|X\|^{2 k}\right)^{1 /(2 k)} \\
& \leq \sup _{k \geq 1} k^{-1 / 2}(2 k)^{1 / 2} \sqrt{\operatorname{tr}(\Sigma) / c_{0}} \\
& \leq \sqrt{2 \operatorname{tr}(\Sigma) / c_{0}}
\end{aligned}
$$

which is the desired result.

Proposition A.2. Let Assumption 1 hold. For any $t \geq 0$,

$$
\mathbb{P}\left\{\|\bar{X}\|^{2} \geq \frac{1+c_{1} t}{n} \cdot \operatorname{tr}(\Sigma)\right\} \leq \exp (1-t),
$$

where $c_{1}=\max \left\{\max \left(\sqrt{C_{*}}, c_{*}\right)\left(4 c_{0}^{-1}+1\right), 2\right\}$ is a constant. Furthermore,

$$
\mathbb{E}\left(\|\bar{X}\|^{4}\right) \leq\left\{1+2\left(c_{1}^{2}+c_{1}\right) \exp (1)\right\} \frac{\operatorname{tr}(\Sigma)^{2}}{n^{2}}
$$

Proof of Proposition A.2. It is straightforward to verify that $\sqrt{n} \bar{X}$ is sub-Gaussian and satisfies Assumption 1 with the same $c_{0}$. Applying the Markov inequality to $\exp \left(n\|\bar{X}\|^{2}\right)$ we obtain, for any $a>0, x \geq c_{*}\left(4 c_{0}^{-1}+1\right) \operatorname{tr}(\Sigma)$,

$$
\begin{aligned}
\mathbb{P}\left\{n\|\bar{X}\|^{2}-\operatorname{tr}(\Sigma) \geq a\right\} & \leq \exp \left(-a t^{-1}\right) \mathbb{E} \exp \left\{x^{-1}\left[n\|\bar{X}\|^{2}-\operatorname{tr}(\Sigma)\right]\right\} \\
& \leq \exp \left(-a x^{-1}\right) \exp \left\{C_{*}\left[\frac{\left(4 c_{0}^{-1}+1\right) \operatorname{tr}(\Sigma)}{x}\right]^{2}\right\},
\end{aligned}
$$

where the last inequality holds by Proposition A.1. By letting $x=c_{1} \operatorname{tr}(\Sigma)$ and $a=t x$ we obtain (A.2).

Next we assume $t \geq \operatorname{tr}(\Sigma)^{2} / n^{2}$ and we have

$$
\begin{aligned}
\mathbb{E}\left(\|\bar{X}\|^{4}\right) & =\int_{0}^{\infty} \mathbb{P}\left(\|\bar{X}\|^{2} \geq \sqrt{y}\right) \mathrm{d} y \\
& \leq t+\int_{t}^{\infty} \exp \left\{1-\frac{n \sqrt{y}-\operatorname{tr}(\Sigma)}{c_{1} \operatorname{tr}(\Sigma)}\right\} \mathrm{d} y \\
& \leq t+2(a \sqrt{t}+1) \exp (b-a \sqrt{t}) / a^{2}
\end{aligned}
$$


where $b=1+c_{1}^{-1}$ and $a^{-1}=c_{1} \operatorname{tr}(\Sigma) / n$. To obtain the second inequality above we employed (A.2). By plugging in $t=\operatorname{tr}(\Sigma)^{2} / n^{2}$ into the above inequalities we obtain the desired result.

Proposition A.3. Let Assumption 1 hold. For all $n \geq 1$ and $t \geq 0$ :

$$
\mathbb{P}\left\{\left\|\Sigma_{n}^{*}-\Sigma\right\|_{F} \geq \frac{2 c_{1}[\sqrt{2 \exp (1)}+8 \sqrt{t}] \cdot \operatorname{tr}(\Sigma)}{\sqrt{n}}\right\} \leq 2 \exp \{-\min (t, 2 \sqrt{n t})\},
$$

where $c_{1}$ is defined in Proposition A.2. Furthermore,

$$
\mathbb{E}\left(\left\|\Sigma_{n}^{*}-\Sigma\right\|_{F}^{2}\right) \leq\left[\frac{4 c_{1} \operatorname{tr}(\Sigma)}{\sqrt{n}}\right]^{2} c_{2}
$$

where $c_{2}=\exp (1)+\int_{0}^{\infty} \exp \left\{-\frac{1}{64} \min (t, 16 \sqrt{t})\right\} \mathrm{d} t$.

Proof of Proposition A.3. By Theorem A.2 the Frobenius norm is 2-smooth on the space $\mathbb{R}^{p \times p}$ of $p \times p$ real matrices. Hence by Proposition A.5 and Theorem A.1,

$$
\mathbb{P}\left\{\left\|\Sigma_{n}^{*}-\Sigma\right\|_{F} \geq \frac{2 c_{1}[\sqrt{2 \exp (1)}+t] \cdot \operatorname{tr}(\Sigma)}{\sqrt{n}}\right\} \leq 2 \exp \left\{-\frac{1}{64} \min \left(t^{2}, 16 t \sqrt{n}\right)\right\}
$$

Inequality (A.3) follows by changing $t$ to $8 \sqrt{t}$ in the above inequality. Denote for the moment $4 c_{1}^{2} \operatorname{tr}(\Sigma)^{2} / n$ by $C_{1}$. Then,

$$
\begin{aligned}
\mathbb{E}\left(\left\|\Sigma_{n}^{*}-\Sigma\right\|_{F}^{2}\right) & \leq 4 \exp (1) C_{1}+\int_{4 \exp (1) C_{1}}^{\infty} \mathbb{P}\left(\left\|\Sigma_{n}^{*}-\Sigma\right\|_{F}^{2} \geq y\right) \mathrm{d} y \\
& \leq 4 \exp (1) C_{1}+2 C_{1} \int_{0}^{\infty} \mathbb{P}\left\{\left\|\Sigma_{n}^{*}-\Sigma\right\|_{F}^{2} \geq 2 C_{1}(t+2 \exp (1))\right\} \mathrm{d} t \\
& \leq 4 \exp (1) C_{1}+2 C_{1} \int_{0}^{\infty} \mathbb{P}\left\{\left\|\Sigma_{n}^{*}-\Sigma\right\|_{F}^{2} \geq C_{1}(\sqrt{t}+\sqrt{2 \exp (1)})^{2}\right\} \mathrm{d} t \\
& \leq 4 \exp (1) C_{1}+4 C_{1} \int_{0}^{\infty} \exp \left\{-\frac{1}{64} \min (t, 16 \sqrt{t n})\right\} \mathrm{d} t \\
& \leq 4 C_{1} c_{2},
\end{aligned}
$$

where $c_{2}=\exp (1)+\int_{0}^{\infty} \exp \left\{-\frac{1}{64} \min (t, 16 \sqrt{t})\right\} \mathrm{d} t$. 
Proposition A.4. Let Assumption 1 hold. For all $n \geq 1$ and $t \geq 0$ :

$$
\mathbb{P}\left\{\left\|\Sigma_{n}^{*}-\Sigma\right\|_{2} \geq c_{3} \cdot\|\Sigma\|_{2} \cdot \max \left\{\sqrt{\frac{r_{e}(\Sigma)(t+\ln p)}{n}}, \frac{r_{e}(\Sigma)(t+\ln p)}{n}\right\}\right\} \leq \exp (-t),
$$

where $c_{3}$ is a fixed constant that depends only on $c_{0}$. Furthermore,

$$
\mathbb{E}\left(\left\|\Sigma_{n}^{*}-\Sigma\right\|_{2}^{2}\right) \leq 5 c_{3}^{2} \cdot\|\Sigma\|_{2}^{2} \cdot \max \left\{\frac{r_{e}(\Sigma) \cdot \ln p}{n},\left(\frac{r_{e}(\Sigma) \cdot \ln p}{n}\right)^{2}\right\} .
$$

Proof of Proposition A.4. Let $Z_{i}=X_{i} X_{i}^{\prime}-\Sigma$, then $\mathbb{E}\left(Z_{i}\right)=0$. We derive that $\Sigma_{n}^{*}=$ $n^{-1} \sum_{i=1}^{n} X_{i} X_{i}^{\prime}=n^{-1} \sum_{i=1}^{n} Z_{i}+\Sigma$ and hence $\left\|\Sigma_{n}^{*}-\Sigma\right\|_{2}=\left\|n^{-1} \sum_{i=1}^{n} Z_{i}\right\|_{2}$. With Proposition A.6, the probability inequality (A.4) is proved by applying Theorem A.3. Next let $\sqrt{y}=3 \cdot \max \left\{\sigma \sqrt{\frac{t+\ln p}{n}}, R \frac{t+\ln p}{n}\right\}$ where $R$ and $\sigma$ are defined in Proposition A.6. Then $t=\min \left(\frac{n y}{9 \sigma^{2}}, \frac{n \sqrt{y}}{3 R}\right)-\ln p$. Let $x=9 \cdot \max \left\{\frac{\sigma^{2} \ln p}{n}, \frac{R^{2}(\ln p)^{2}}{n^{2}}\right\}$. First assume $\sigma^{2} / R^{2} \geq \ln p / n$, then $x=9 \sigma^{2} \ln p / n$ and

$$
\begin{aligned}
\mathbb{E}\left(\left\|\Sigma_{n}^{*}-\Sigma\right\|_{2}^{2}\right) & \leq x+\int_{x}^{\infty} \mathbb{P}\left\{\left\|\Sigma_{n}^{*}-\Sigma\right\|_{2}^{2} \geq y\right\} \mathrm{d} y \\
& \leq x+\int_{x}^{\infty} \exp \left\{-\min \left(\frac{n y}{9 \sigma^{2}}, \frac{n \sqrt{y}}{3 R}\right)+\ln p\right\} \mathrm{d} y \\
& \leq x+p \int_{x}^{\left(3 \sigma^{2} / R\right)^{2}} \exp \left(-\frac{n y}{9 \sigma^{2}}\right) \mathrm{d} y+p \int_{\left(3 \sigma^{2} / R\right)^{2}}^{\infty} \exp \left(-\frac{n \sqrt{y}}{3 R}\right) \mathrm{d} y \\
& \leq x+9 \sigma^{2} / n-9 \sigma^{2} p \exp \left(-n \sigma^{2} / R^{2}\right) / n+18 p \exp \left(-n \sigma^{2} / R^{2}\right)\left(\sigma^{2}+R^{2} / n\right) / n \\
& \leq 36 \sigma^{2} \ln p / n .
\end{aligned}
$$

In the above derivation, we used the assumption $\sigma^{2} / R^{2} \geq \ln p / n$ as well as the inequality $p \exp \left(-n \sigma^{2} / R^{2}\right) \leq 1$ which follows by the assumption. Next assume $\sigma^{2} / R^{2} \leq \ln p / n$, then $x=9 R^{2}(\ln p)^{2} / n^{2}$. It is easy to show that

$$
\begin{aligned}
\mathbb{E}\left(\left\|\Sigma_{n}^{*}-\Sigma\right\|_{2}^{2}\right) & \leq x+p \int_{x}^{\infty} \exp \left(-\frac{n \sqrt{y}}{3 R}\right) \mathrm{d} y \\
& \leq x+18 R^{2}(\ln p+1) / n^{2} \\
& \leq 45 R^{2}(\ln p)^{2} / n^{2}
\end{aligned}
$$


Therefore, we have, in either case,

$$
\mathbb{E}\left(\left\|\Sigma_{n}^{*}-\Sigma\right\|_{2}^{2}\right) \leq 45 \cdot \max \left\{\frac{\sigma^{2} \ln p}{n}, \frac{R^{2}(\ln p)^{2}}{n^{2}}\right\}
$$

and the proof is complete.

Proposition A.6. Let Assumption 1 hold, and define $Z=X X^{\prime}-\Sigma$, where $\Sigma$ is the covariance matrix of $X$. Let $\tilde{c}_{1}=\sup _{d \geq 1} \exp (-d) d^{d} / d$ !, $\tilde{c}_{2}=\tilde{c}_{1} c_{0}^{2} \exp (-1)+\tilde{c}_{1} \exp (-1) / 4+3$ and $\tilde{c}_{3}=\max \left\{4 \exp (1) / c_{0}, 1\right\}$. If we let $R=2 \tilde{c}_{3} \cdot \operatorname{tr}(\Sigma)$ and $\sigma^{2}=\tilde{c}_{2} \tilde{c}_{3}^{2} \cdot \operatorname{tr}(\Sigma) \cdot\|\Sigma\|_{2}$, then

$$
\left\|\mathbb{E}\left(Z^{d}\right)\right\|_{2} \leq \frac{d !}{2} \cdot R^{d-2} \sigma^{2} \quad \text { for } d=2,3, \ldots
$$

Proof of Proposition A.6. Let $u \in \mathbb{R}^{p}$ be a unit vector. First, by Lemma A.5 below,

$$
u^{\prime} Z^{d} u \leq\|Z\|_{2}^{d-1}\left\{u^{\prime}\left(X X^{\prime}+2\|\Sigma\|_{2} \cdot I_{p}\right) u\right\}=\|Z\|_{2}^{d-1}\left\{\left(u^{\prime} X\right)^{2}+2\|\Sigma\|_{2}\right\} .
$$

Next since $\|Z\|_{2} \leq\left\|X X^{\prime}\right\|_{2}+\|\Sigma\|_{2} \leq\|X\|^{2}+\|\Sigma\|_{2}$, we derive that

$$
\|Z\|_{2}^{d-1} \leq\left(\|X\|^{2}+\|\Sigma\|_{2}\right)^{d-1} \leq 2^{d-2}\left(\|X\|^{2(d-1)}+\|\Sigma\|_{2}^{d-1}\right)
$$

Equations (A.5) and (A.6) together imply

$$
u^{\prime} Z^{d} u \leq 2^{d-2}\left(\|X\|^{2(d-1)}+\|\Sigma\|_{2}^{d-1}\right)\left\{\left(u^{\prime} X\right)^{2}+2\|\Sigma\|_{2}\right\}
$$

Hence

$$
\mathbb{E}\left(u^{\prime} Z^{d} u\right) \leq 2^{d-2} \mathbb{E}\left\{\|X\|^{2(d-1)}\left(u^{\prime} X\right)^{2}+2\|X\|^{2(d-1)}\|\Sigma\|_{2}+\|\Sigma\|_{2}^{d-1}\left(u^{\prime} X\right)^{2}+2\|\Sigma\|_{2}^{d}\right\}
$$

By Assumption $1,\|\Sigma\|_{2} \geq c_{0}\left(\mathbb{E}\left\|u^{\prime} X\right\|^{2 d}\right)^{1 / d} /(2 d)$ for any positive integer $d$, i.e., $\mathbb{E}\left\|u^{\prime} X\right\|^{2 d} \leq$ $c_{0}^{d}(2 d)^{d}\|\Sigma\|_{2}^{d}$. Then

$$
\begin{aligned}
\mathbb{E}\left\{\|X\|^{2(d-1)}\left(u^{\prime} X\right)^{2}\right\} & \leq \sqrt{\mathbb{E}\|X\|^{4(d-1)} \cdot \mathbb{E}\left(u^{\prime} X\right)^{4}} \\
& \leq \sqrt{\mathbb{E}\|X\|^{4(d-1)} \cdot c_{0}^{2} 4^{2}\|\Sigma\|_{2}^{2}} \\
& \leq 4 c_{0}\|\Sigma\|_{2} \sqrt{\mathbb{E}\|X\|^{4(d-1)}}
\end{aligned}
$$


By Lemma A.2 above, we further derive from (A.7) and (A.8) that

$$
\begin{aligned}
\mathbb{E}\left(u^{\prime} Z^{d} u\right) & \leq 2^{d-2}\left\{4 c_{0}\|\Sigma\|_{2}(4 d-4)^{d-1} \operatorname{tr}(\Sigma)^{d-1} / c_{0}^{d-1}+2(2 d-2)^{d-1} \operatorname{tr}(\Sigma)^{d-1}\|\Sigma\|_{2} / c_{0}^{d-1}+3\|\Sigma\|_{2}^{d}\right\} \\
& \leq 2^{d-2}\left\{\|\Sigma\|_{2} \operatorname{tr}(\Sigma)^{d-1}\right\}\left\{4 c_{0}(4 d-4)^{d-1} / c_{0}^{d-1}+2(2 d-2)^{d-1} / c_{0}^{d-1}+3\right\} \\
& \leq 2^{d-2}\left\{\|\Sigma\|_{2} \operatorname{tr}(\Sigma)^{d-1}\right\} \max \left\{4 \exp (1) / c_{0}, 1\right\}^{d} d !\left\{\tilde{c}_{1} c_{0}^{2} \exp (-1) / d+\tilde{c}_{1} 2^{-d} \exp (-1) / d+3 / d !\right\} \\
& \leq 2^{d-2} d ! \max \left\{4 \exp (1) / c_{0}, 1\right\}^{d} d !\left\{\|\Sigma\|_{2} \operatorname{tr}(\Sigma)^{d-1}\right\}\left\{\tilde{c}_{1} c_{0}^{2} \exp (-1) / 2+\tilde{c}_{1} \exp (-1) / 8+3 / 2\right\}
\end{aligned}
$$

where $\tilde{c}_{1}=\sup _{d \geq 1} \exp (-d) d^{d} / d$ !. Because the above inequality holds for any unit vector $u$,

$$
\left\|\mathbb{E}\left(Z^{d}\right)\right\|_{2} \leq \tilde{c}_{2} 2^{d-3} d ! \max \left\{4 \exp (1) / c_{0}, 1\right\}^{d}\left\{\|\Sigma\|_{2} \operatorname{tr}(\Sigma)^{d-1}\right\}
$$

where $\tilde{c}_{2}=\tilde{c}_{1} c_{0}^{2} \exp (-1)+\tilde{c}_{1} \exp (-1) / 4+3$. The proof is complete.

Lemma A.4. Suppose $A, B \in \mathbb{R}^{p \times p}$ are two positive semi-definite matrices. Let $O D O^{\prime}$ be an eigendecomposition of $A-B$ with $D=\operatorname{diag}\left(\lambda_{1}, \ldots, \lambda_{p}\right)$. Let $D^{+}=\operatorname{diag}\left(\left|\lambda_{1}\right|, \ldots,\left|\lambda_{p}\right|\right)$. Then $O D^{+} O^{\prime} \leq A+2\|B\|_{2} \cdot I_{p}$, where the notation " $\leq$ " was used to compare two matrices and for two matrices $E_{1}$ and $E_{2}, E_{1} \leq E_{2}$ implies $E_{2}-E_{1}$ is psd.

Proof of Lemma $A .4$. Let $u_{k}$ be the $k$-th column of $O$, then $\lambda_{k}=u_{k}^{\prime}(A-B) u_{k} \geq-\|B\|_{2}$. This implies if $\lambda_{k}$ is negative, $\left|\lambda_{k}\right| \leq\|B\|_{2}$. Hence $\left|\lambda_{k}\right|-\lambda_{k} \leq 2\|B\|_{2}$ and $D^{+} \leq D+2\|B\|_{2} \cdot I_{p}$. It follows that $O D^{+} O^{\prime} \leq O\left(D+2\|B\|_{2} \cdot I_{p}\right) O^{\prime}=A-B+2\|B\|_{2} \cdot I_{p} \leq A+2\|B\|_{2} \cdot I_{p}$.

Lemma A.5. Suppose $A, B \in \mathbb{R}^{p \times p}$ are two positive semi-definite matrices. Fix $u \in \mathbb{R}^{p}$. For an arbitrary positive integer d,

$$
u^{\prime}(A-B)^{d} u \leq\|A-B\|_{2}^{d-1}\left\{u^{\prime}\left(A+2\|B\|_{2} \cdot I_{p}\right) u\right\}
$$

Proof of Lemma A.5. Let $O D O^{\prime}$ be an eigendecomposition of $A-B$ with $D=\operatorname{diag}\left(\lambda_{1}, \ldots, \lambda_{p}\right)$ and define $D^{+}=\operatorname{diag}\left(\left|\lambda_{1}\right|, \ldots,\left|\lambda_{p}\right|\right)$. Let $\tilde{u}=O^{\prime} u$. Then $u^{\prime}(A-B)^{d} u=\left(O^{\prime} u\right)^{\prime} D^{d}\left(O^{\prime} u\right)=$ $\tilde{u}^{\prime} D^{d} \tilde{u}=\sum_{j=1}^{p} \lambda_{j}^{d} \tilde{u}_{j}^{2} \leq \max _{j}\left|\lambda_{j}\right|^{d-1} \cdot \sum_{j=1}^{p}\left|\lambda_{j}\right| \tilde{u}_{j}^{2}=\|A-B\|_{2}^{d-1}\left(u^{\prime} O D^{+} O^{\prime} u\right)$, i.e., $u^{\prime}(A-$ $B)^{d} u \leq\|A-B\|_{2}^{d-1}\left(u^{\prime} O D^{+} O^{\prime} u\right)$. By Lemma A.4, $O D^{+} O^{\prime} \leq A+2\|B\|_{2} \cdot I_{p}$ and the proof is complete. 
Theorem A.4. Suppose $X$ is a random vector that satisfies Assumption 1. Let $n>1$. If $\Sigma \in \mathcal{P}_{1}(\epsilon)$, then with probability $1-11 n^{-1}$,

$$
\left|\frac{r_{e}\left(\Sigma_{n}\right)}{r_{e}(\Sigma)}-1\right| \lesssim \max \left\{\sqrt{\frac{r_{e}(\Sigma) \cdot \ln p n}{2 n}}, \frac{r_{e}(\Sigma) \cdot \ln p n}{n}\right\} ;
$$

If $\Sigma \in \mathcal{P}_{2}(\epsilon)$, then with probability $1-11 n^{-1}$,

$$
\left|\frac{r_{e}\left(\Sigma_{n}\right)}{r_{e}(\Sigma)}-1\right| \lesssim \frac{r_{e}(\Sigma) \cdot \ln n}{n} .
$$

Proof of Theorem A.4. Let $c_{j}, j=1,2,3$ be defined as in Propositions A.2, A.3 and A.4. We will only prove the case $\Sigma \in \mathcal{P}_{1}(\epsilon)$ because the proof for the other case is similar. First we have

$$
\begin{aligned}
\left|\frac{r_{e}\left(\Sigma_{n}\right)}{r_{e}(\Sigma)}-1\right| & =\left|\frac{\operatorname{tr}\left(\Sigma_{n}\right)}{\operatorname{tr}(\Sigma)} \cdot \frac{\|\Sigma\|_{2}}{\left\|\Sigma_{n}\right\|_{2}}-1\right| \\
& \leq\left|\frac{\operatorname{tr}\left(\Sigma_{n}\right)-\operatorname{tr}(\Sigma)}{\operatorname{tr}(\Sigma)}\right| \cdot \frac{\|\Sigma\|_{2}}{\left\|\Sigma_{n}\right\|_{2}}+\left|\frac{\|\Sigma\|_{2}}{\left\|\Sigma_{n}\right\|_{2}}-1\right| .
\end{aligned}
$$

Let

$$
a_{1}=2 c_{1}\left\{\frac{\ln n}{n}+\sqrt{\frac{\ln n}{n}}\right\}
$$

and

$$
a_{2}=\left(c_{1}+c_{3}+1\right) \cdot \max \left\{\sqrt{\frac{r_{e}(\Sigma) \cdot \ln p n}{n}}, \frac{r_{e}(\Sigma) \cdot \ln p n}{n}\right\} .
$$

Then by Theorems 2.2 and 2.3 , with probability at least $1-11 n^{-1}$,

$$
\left|\operatorname{tr}\left(\Sigma_{n}\right)-\operatorname{tr}(\Sigma)\right| \leq a_{1} \operatorname{tr}(\Sigma)
$$

and

$$
\left\|\Sigma_{n}-\Sigma\right\|_{2} \leq a_{2}\|\Sigma\|_{2} .
$$

We derive from equation (A.10) that

$$
\frac{1}{1+a_{2}} \leq \frac{\|\Sigma\|_{2}}{\left\|\Sigma_{n}\right\|_{2}} \leq \frac{1}{1-a_{2}},
$$


and furthermore

$$
\left|\frac{\|\Sigma\|_{2}}{\left\|\Sigma_{n}\right\|_{2}}-1\right| \leq \frac{a_{2}}{1-a_{2}} .
$$

It follows by inequality (A.9) that

$$
\left|\frac{r_{e}\left(\Sigma_{n}\right)}{r_{e}(\Sigma)}-1\right| \leq \frac{a_{1}}{1-a_{2}}+\frac{a_{2}}{1-a_{2}}=\frac{a_{1}+a_{2}}{1-a_{2}}
$$

and hence the theorem follows.

Lemma A.6. Suppose the assumptions in Proposition 5.1 hold. Let $A=\left[\phi_{1}, \ldots, \phi_{N}\right]$ be an $m \times N$ matrix. Let $(Q, R)$ be a $Q R$ decomposition of $A$ where $Q$ is an $m \times N$ matrix with orthonormal columns and $R$ is an $N \times N$ upper-triangular matrix. Denote the $(k, j)$ th element of $R$ by $R_{k j}$. Let $N$ be a positive integer such that $12 C_{7 \lambda} N^{1+\gamma_{1}} \leq m$ where $C_{7 \lambda}$ is the constant as in inequality (A.6) of Proposition 5.1. If A has full rank, then there exists a pair of $Q$ and $R$ such that if $k>j, R_{k j}=0$ and if $k \leq j$,

$$
\left|R_{k j}-\delta_{k, j}-\delta_{k, j} r_{k}\right| \leq 3 C_{7 \lambda} j^{\gamma_{1}} / m
$$

where $r_{k}$ is defined in such a way that for all $k \leq N$

$$
\left|r_{k}\right| \leq 4 C_{7 \lambda} k^{1+\gamma_{1}} / m
$$

Proof of Lemma A.6. We construct $Q$ and $R$ by the Gram-Schmidt process. Let $\mathbf{u}_{1}=$ $\phi_{1}, \mathbf{v}_{1}=\mathbf{u}_{1} /\left\|\mathbf{u}_{1}\right\|$. For $k=2, \ldots, N$, define $\mathbf{u}_{k}=\phi_{k}-\sum_{j=1}^{k-1}\left(\boldsymbol{\phi}_{k}^{\prime} \mathbf{v}_{j}\right) \mathbf{v}_{j}, \mathbf{v}_{k}=\mathbf{u}_{k} /\left\|\mathbf{u}_{k}\right\|$ and $r_{k}=1 /\left\|\mathbf{u}_{k}\right\|-1$. We let $Q=\left[\mathbf{v}_{1}, \ldots, \mathbf{v}_{N}\right]$ and $R=Q^{\prime} A$. Denote $R_{k j}$ the $(k, j)$ th element of $R$. Then $R_{k j}=\phi_{j}^{\prime} \mathbf{v}_{k}$. Note that $\left\{\mathbf{v}_{1}, \ldots, \mathbf{v}_{N}\right\}$ are orthonormal vectors and $\phi_{k}$ can be written as a linear combination of $\left\{\mathbf{v}_{1}, \ldots, \mathbf{v}_{k}\right\}$. Hence for $k>j, R_{k j}=0$ and for $k \leq j$, $R_{k j}=\left(\phi_{j}^{\prime} \mathbf{u}_{k}\right) /\left\|\mathbf{u}_{k}\right\|$. Because

$$
\left|R_{k j}-\delta_{k, j}-\delta_{k, j} r_{k}\right|=\frac{\left|\phi_{j}^{\prime} \mathbf{u}_{k}-\delta_{k, j}\right|}{\left\|\mathbf{u}_{k}\right\|},
$$

the lemma is proved if we can show that for all $k \leq N$

$$
|| \mathbf{u}_{k} \|-1 \mid \leq 3 C_{7 \lambda} k^{1+\gamma_{1}} / m
$$


and

$$
\left|\boldsymbol{\phi}_{j}^{\prime} \mathbf{u}_{k}-\delta_{j, k}\right| \leq \frac{2 C_{7 \lambda} j^{\gamma_{1}}}{m} \text { for all } j \text { with } k \leq j \leq N
$$

where $C_{7 \lambda}$ is the fixed constant in (A.6) of Proposition 5.1. In particular, the inequality $\left|r_{k}\right| \leq 4 C_{7 \lambda} k^{1+\gamma_{1}} / m$ can be proved by (A.11).

We prove equalities (A.11) and (A.12) by induction on the range of $k$. For $k=1, \mathbf{u}_{1}=\phi_{1}$. Hence $\left\|\mathbf{u}_{k}\right\|=\left\|\phi_{1}\right\|$ and (A.11) holds for $k=1$ by inequality (A.6) of Proposition 5.1. Inequality (A.12) for $k=1$ can also be proved by (A.6) of Proposition 5.1. Assume (A.11) and (A.12) hold for all $k \leq N_{0}-1$ with $N_{0} \leq N$, then we need to prove that

$$
||\left|\mathbf{u}_{N_{0}} \|-1\right| \leq 3 C_{7 \lambda} N_{0}^{1+\gamma_{1}} / m
$$

and

$$
\left|\phi_{j}^{\prime} \mathbf{u}_{N_{0}}-\delta_{j, N_{0}}\right| \leq \frac{2 C_{7 \lambda} j^{\gamma_{1}}}{m} \text { for all } j \text { with } N_{0} \leq j \leq N
$$

We first prove (A.13). By definition of $\mathbf{u}_{N_{0}}$,

$$
\left\|\mathbf{u}_{N_{0}}-\phi_{N_{0}}\right\| \leq \sum_{k=1}^{N_{0}-1}\left|\phi_{N_{0}}^{\prime} \mathbf{v}_{k}\right| \leq \sum_{k=1}^{N_{0}-1} \frac{\left|\boldsymbol{\phi}_{N_{0}}^{\prime} \mathbf{u}_{k}\right|}{\left\|\mathbf{u}_{k}\right\|} \leq \frac{2 C_{7 \lambda} N_{0}^{1+\gamma_{1}}}{m\left(1-3 C_{7 \lambda} N_{0}^{1+\gamma_{1}} / m\right)}
$$

by the induction assumption. Since $\left|\left\|\phi_{N_{0}}\right\|^{2}-1\right| \leq C_{7 \lambda} N_{0}^{\gamma_{1}} / m$ by inequality (A.6) of Proposition 5.1, we can derive $\left|\left\|\phi_{N_{0}}\right\|-1\right| \leq \frac{C_{7 \lambda} N_{0}^{\gamma_{1}}}{2 m \sqrt{1-C_{7 \lambda} N_{0}^{\gamma_{1}} / m}}$ and hence

$$
\begin{aligned}
\left|\left\|\mathbf{u}_{N_{0}}\right\|-1\right| & \leq\left|\left\|\mathbf{u}_{N_{0}}\right\|-\left\|\phi_{N_{0}}\right\|\right|+\left|\left\|\phi_{N_{0}}\right\|-1\right| \\
& \leq \frac{2 C_{7 \lambda} N_{0}^{1+\gamma_{1}}}{m\left(1-3 C_{7 \lambda} N_{0}^{1+\gamma_{1}} / m\right)}+\frac{C_{7 \lambda} N_{0}^{\gamma_{1}}}{2 m \sqrt{1-C_{7 \lambda} N_{0}^{\gamma_{1}} / m}} .
\end{aligned}
$$

It follows that

$$
||\left|\mathbf{u}_{N_{0}} \|-1\right| \leq \frac{2 C_{7 \lambda} N_{0}^{1+\gamma_{1}}}{m\left(1-3 C_{7 \lambda} N_{0}^{1+\gamma_{1}} / m\right)}+\frac{C_{7 \lambda} N_{0}^{\gamma_{1}}}{2 m \sqrt{1-C_{7 \lambda} N_{0}^{\gamma_{1}} / m}} \leq 3 C_{7 \lambda} N_{0}^{1+\gamma_{1}} / m
$$

by the assumption that $12 C_{7 \lambda} N^{1+\gamma_{1}} / m \leq 1$. So we have proved (A.13).

We now prove (A.14). Note that for $j \geq N_{0}$,

$$
\phi_{j}^{\prime} \mathbf{u}_{N_{0}}=\phi_{j}^{\prime} \boldsymbol{\psi}_{N_{0}}-\sum_{k=1}^{N_{0}-1}\left(\phi_{N_{0}}^{\prime} \mathbf{v}_{k}\right)\left(\phi_{j}^{\prime} \mathbf{v}_{k}\right)
$$


By the induction assumption, for $k \leq N_{0}-1,\left|\phi_{N_{0}}^{\prime} \mathbf{v}_{k}\right|=\left|\phi_{N_{0}}^{\prime} \mathbf{u}_{k}\right| /\left\|\mathbf{u}_{k}\right\| \leq 3 C_{7 \lambda} N_{0}^{\gamma_{1}} / m$ by the assumption on $N$. Similarly, $\left|\boldsymbol{\phi}_{j}^{\prime} \mathbf{v}_{k}\right| \leq 3 C_{7 \lambda} j^{\gamma_{1}} / m$. Hence $\left|\phi_{j}^{\prime} \mathbf{u}_{N_{0}}-\boldsymbol{\phi}_{j}^{\prime} \boldsymbol{\phi}_{N_{0}}\right| \leq$ $9 C_{7 \lambda}^{2} N_{0}^{1+\gamma_{1}} j^{\gamma_{1}} / m^{2} \leq 3 C_{7 \lambda} j^{\gamma_{1}} /(4 m)$ and

$$
\left|\phi_{j}^{\prime} \mathbf{u}_{N_{0}}-\delta_{j, N_{0}}\right| \leq\left|\phi_{j}^{\prime} \mathbf{u}_{N_{0}}-\phi_{j}^{\prime} \phi_{N_{0}}\right|+\left|\phi_{j}^{\prime} \phi_{N_{0}}-\delta_{j, N_{0}}\right| \leq 3 C_{7 \lambda} j^{\gamma_{1}} /(4 m)+C_{7 \lambda} / m \leq 2 C_{7 \lambda} j^{\gamma_{1}}
$$

which proves (A.14) and also the lemma.

\section{References}

[1] Bunea, F. and Xiao, L. (2014). On the sample covariance matrix estimator of reduced effective rank population matrices, with applications to fPCA. 